\title{
SOBRE FENOMENOLOGÍA DEL CONOCIMIENTO
}

\author{
Milan Marinovic $\mathrm{P}^{*}$
}

\section{CONTEXTO}

El proceso de modernismo, que conlleva el desarrollo científico y tecnológico, sitúa al ser humano frente a su devenir histórico en una actitud distinta que en la dinámica de cambios. Esto se hace perceptible a través de las dimensiones sociales y sus funciones primarias en lo que Teilhard de Chardin define como visión de cosmogénesis.

La visión de un universo evolutivo, que descorre el racionalismo científico y ante el cual el ser humano se percibe como artífice, contrasta con la visión de un mundo cósmico que situaba al ser humano en actitud contemplativa.

En su dimensión energética, la visión de cosmogénesis percibe la naturaleza como un lugar de vida de las especies orgánicas. Emerge así la concepción de ecosistema, que interpretando la calidad de vida en su relación con el medio, valora las condiciones que la favorece y hacen posible el equilibrio ecológico como una función primaria de preservación que promueve las condiciones de seguridad de vida humana y de las especies.

En su dimensión estructural, los avances del desarrollo científico en el campo de las comunicaciones e informática, rompen el esquema perceptual del mundo cósmico y la imagen de "lejano planeta", que en la visión de cosmogénesis adquiere ahora el tamaño de una aldea en la que la humanidad entera se percibe como vecino y solidario.

* El autor, miembro de la Facultad de Ciencias Políticas y Sociología de la Universidad de Salamanca, comenta y critica en este trabajo el artículo "Fenomenología del Conocimiento" de Humberto Maturana, Revista de Tecnología Educativa vol. 8, 1983, de la U. de Chile. 
Emergen entonces los acuerdos, los tratados y leyes explícitos que regulan y norman las interacciones entre los Estados y sus poblaciones, ello como función primaria de integración que promueve las condiciones de solidaridad social, requisito de la convivencia humana.

Finalmente, en su dimensión funciona/ la visión de cosmogénesis nos muestra un mundo que se organiza en la satisfacción de sus necesidades colectivas que emergiendo de las necesidades primarias de preservación e integración buscan primero la coexistencia pacífica entre los Estados, a la vez que la integración de una economía mundial y luego el acuerdo de las Naciones, como una función primaria de desarrollo que promueve las condiciones del progreso humano.

En este contexto, la visión de un mundo cósmico sitúa al ser humano en contradicción al proceso de modernismo, con un criterio de validación que opera en un mundo de recursos ilimitados que pueden ser apropiados competitivamente. El resultado es la riqueza de unos y la miseria de muchos en un continuo agotamiento del mundo natural, que no es infinito.

Ante la irracionalidad de las guerras, la continua creación de miseria en la aparente abundancia, la explotación indiscriminada de los recursos, la depredación de las especies y la irracionalidad de la conducta que contamina el ambiente o lugar de vida, surge como consecuencia de la visión de cosmogénesis en la humanidad una actitud contestataria que como valor colectivo, en el plano de la trascendencia unos y en el de la inmanencia otros, valora la vida y las condiciones de convivencia mutua que la aseguran y generan.

Frente al dilema existencial que conlleva la percepción de dos mundos y como una reacción a la quimera de la indiferencia, el autor de la Fenomenología del Conocer, en un estudio exploratorio, proporciona un criterio de validación que lo resuelve y abre un espacio en la filosofía pragmática de la coexistencia humana.

\section{SINTESIS}

\section{A la Introducción}

El conocimiento es un fenómeno de naturaleza biológica, el cual se revela a un sujeto en su ocurrir cotidiano a partir del fenómeno de la percepción. El ser humano, en su diario vivir y desde su perspectiva, va rellenando huecos o vacíos perceptuales que se generan dimensionalmente en su estructura anatómica conductual y cultural.

El conocer en el plano de sus dimensiones conlleva, para el sujeto, interrogantes que definen el ámbito de sus respuestas y que se satisfacen desde la perspectiva de quien lo genera.

Frente al fenómeno del conocimiento la pregunta que genera un sujeto define el ámbito o el dominio cognoscitivo de la respuesta que lo ha de satisfacer. Para que la respuesta satisfaga al sujeto y sea aceptada por ésta como verdadera -aparte de 
darse en el dominio definido- la pregunta debe satisfacer un criterio de validación aceptado por el sujeto en cuyo caso la respuesta constituirá en la percepción del sujeto una acción o conducta efectiva.

Son dominios cognoscitivos todo campo del saber que legitimado por un criterio de validación conductual establece normas en la interacción (el campo de las ciencias, la religión, los juegos, etc.).

Conocimiento en consecuencia es la acción o conducta efectiva que satisface la interrogante planteada por un sujeto en el dominio cognoscitivo o ámbito de su pregunta.

Inferencias:

Para determinar un conocimiento aplicamos en el poceso de la interacción el criterio de conducta efectiva en un determinado campo o dominio que el sujeto especifica en su pregunta.

Conocer no es saber cosas, no es resolver problemas, conocer es tener una conducta efectiva en el dominio cognoscitivo o ámbito especificado por el sujeto en su pregunta.

Frente al fenómeno del conocimiento, en el proceso de interacción hay que definir en consecuencia cuáles son las condiciones existenciales que aceptamos como conducta efectiva. Esto es, cual es el criterio de validación que en nuestro diario vivir aplicamos para definir una conducta efectiva en cualquier ámbito o dominio en que se especifique una pregunta.

El criterio de la conducta efectiva que constituye la tesis del estudio -es que los seres vivos son sistemas que interactúan en condiciones de adaptación y conservación determinados por su estructura.

\section{A la Metodologia}

Como método heurístico el autor define los conceptos de unidad, organización y estructura que son constitutivos de un sistema.

En el diseño de su hipótesis explicativa, Maturana aplica estos conceptos a los sistemas vivos, demuestra que los seres humanos, comunidades compuestas con organización y estructura, son sistemas autónomos que se producen a sí mismos y que determinan por su estructura, en qué ámbito pueden interactuar conservando su adaptación y organización.

\section{Definiciones:}

Unidad: se especifica por un criterio de distinción que diferencia unidades simples y compuestas. 
a) Unidades simples: son distinciones genéricas que identifican por su función a un ente o ítem cultural (silla, vehículo, pastel, etc.).

b) Unidades compuestas: son unidades simples diferenciadas por su estructura (silla Luis XV, autobús, pastel de chocolate, etc.).

Toda unidad compuesta se define por la identidad de su función organizacional y se organiza por su estructura.

Organización: se define por su función de identidad de clase en una unidad compuesta (sentarse, movilizarse, alimentarse).

La organización de una unidad es invariante mientras conserve su identidad.

Si cambia la organización, la unidad pierde su identidad.

Todo sistema se desintegra cuando la organización que lo define pierde su identidad.

Estructura: se define como el instrumento que realiza la función organizacional.

En tanto la organización a través de su función se mantiene constante, la estructura puede variar.

Sólo la dinámica estructural genera cambios en un sistema sin perder su identidad.

Inferencias:

Los sistemas organizacionales son unidades compuestas que se componen de estructura y organización.

Los sistemas organizacionales se determinan por su estructura, al ser éste el único elemento que puede variar sin perder su función organizacional.

La dinámica estructural se expresa en sus dimensiones sociales a través de los cambios anatómicos conductuales y culturales.

Sólo la dinámica estructural genera cambios y en ella se basa la hipótesis explicativa como mecanismo que genera el fenómeno a demostrar.

La ciencia en el campo de la investigación, sólo puede tratar con sistemas que estén determinados por sus estructuras al ser la dinámica estructural el único mecanismo de la hipótesis explicativa capaz de generar el fenómeno.

Solamente en la medida que tratemos a los seres humanos como sistemas vivos determinados por su estructura podemos analizarlos en la ciencia.

\section{A la hipótesis explicativa (aplicada a los sistemas vivos)}

Los seres humanos como sistemas vivos son unidades compuestas que en su dinámica de estados están determinados por la estructura de sus relaciones.

Desde el punto de vista científico, los seres humanos son sistemas abiertos en continua transformación orgánica conservando su organización en tanto conservan su identidad como tal. 
Lo particular de los sistemas vivos es su organización autopoiética, que los define como sistemas moleculares autónomos en tanto ocurren en éstos un continuo cambio estructural.

Los seres humanos, y en general todo sistema vivo con organización y estructu$r a$, existen en un medio de interacciones sólo en tanto conserven su capacidad de adaptación y por esa vía su organización.

Las variables externas en el proceso de interacción pueden ser de naturaleza perturbadora en cuyo caso desencadenan en el sistema un cambio de estado o de naturaleza destructiva que amenazan su desintegración.

Todo ser vivo interactúa en un continuo cambio estructural, adaptándose a las variables de su entorno para la conservación de su organización, para lograr la "realización de su nicho".

Inferencias:

Los seres vivos son sistemas que determinan con su estructura el ámbito en el que interactúan con conservación de su organización y adaptación.

Todo sistema vivo existe siempre en correspondencia con su medio en el cual interactúa o se desintegra.

El curso que siguen los cambios estructurales de un sistema dinámico que conserva su organización en un contexto determinado es contingente a sus interacciones en la "realización de su nicho".

Para un ser vivo todas sus interacciones son fundamentales porque en sus secuencias selecciona el curso de sus estructuras en un proceso que tiene carácter histórico e irreversible.

Ningún ser vivo está donde está por accidente, sino como resultado de la historia de sus interacciones.

El fenómeno del existir humano no es un fenómeno trivial. Es un fenómeno en el cual nuestras estructuras: anatómica, conductual y cultural están en continuo cambio, adaptándose en la satisfacción de las necesidades que se derivan de sus funciones primarias de preservación, integración y desarrollo. Su interactuar lo hace de manera contingente a las estructuras y necesidades primarias de los otros sistemas con quienes interactúan en un mismo contexto.

El criterio de conducta efectiva que en nuestro diario vivir aplicamos en la interacción emerge desde la perspectiva de la organización y adaptación del ser vivo como sistema en el desarrollo de sus funciones primarias.

III. CRITICA

Si consideramos que la fenomenología, como escuela del pensamiento sociológico, es un estudio sistemático de una teoría de la subjetividad de los fenómenos, cuyo último fin consiste en lograr la clarificación o elucidación de éstos, la fenome- 
nología del conocimiento en los términos propuestos por el autor, tanto por su metodología de análisis, en la explicación científica, como por las premisas que se infieren de su contenido, constituye un aporte significativo en el campo de la teoría fenomenológica para el esclarecimiento del fenómeno del conocer.

La motivación del autor tanto por su planteamiento teórico y como por sus premisas, que se derivan de la hipótesis explicativa, se infieren de las condiciones existenciales que caracterizan la época moderna, cuyo criterio de validación confronta la humanidad al dilema ético que conlleva a un mundo competitivo frente a una continua y creciente creación de miseria en la aparente abundancia. Igualmente se infiere del interés científico que, en el campo de su dominio cognoscitivo, la biología sugiere al autor la constante de la existencia orgánica en la que todo ser viviente interactúa y existe en su medio solamente en la medida que conserva su organización y adaptación.

Frente al fenómeno del conocimiento y que el autor define en términos existenciales en función de la percepción del sujeto, el estudio plantea como tesis el criterio de validación para legitimar una conducta efectiva en la interacción.

El marco filosófico que regula el análisis del autor en relación al fenómeno del conocer se inserta en los principios de la fenomenología establecidos por Edmund Husserl, quien puso de manifiesto la naturaleza dual de la conciencia al distinguir un objeto real de uno mentado.

A la luz de la reflexión fenomenológica de Husserl, el objeto tal y como es en realidad resulta ser también el objeto tal y como aparece al sujeto.

En este contexto la fenomenología del conocer de Maturana se enmarca en e! concepto de nóema de Husserl quien así lo define para distinguir el objeto tal y como es mentado en el acto intencional.

Asimismo, en la misma línea del discurrir teórico Aaron Gurwitch, en su análisis fenomenológico de E/ campo de la Conciencia, establece la relación de identidades entre los conceptos de nóema de Husseri y asunto del pensamiento de William James, para diferenciar el objeto real del mentado en el acto intencionado.

Establecida la relación entre Husserl, considerado el padre de la fenomenología, y William James, considerado el padre de la filosofía pragmática, por la explicación biológica conductista que hace de los fenómenos sicológicos, podemos concluir:

- El aporte teórico de la fenomenología del conocimiento por su marco teórico fundamentado en la filosofía de Husserl y su adaptación orgánica inspirada en el principio biológico del evolucionismo de Darwin, es el hecho que como estudio exploratorio constituye una expresión fenomenológica de la filosofía pragmática.

- Más significativo aún del aporte al autor, es que proporciona un criterio de validación en el proceso de interacción para identificar la conducta efectiva que define el conocimiento que abre un espacio a la coexistencia humana. 